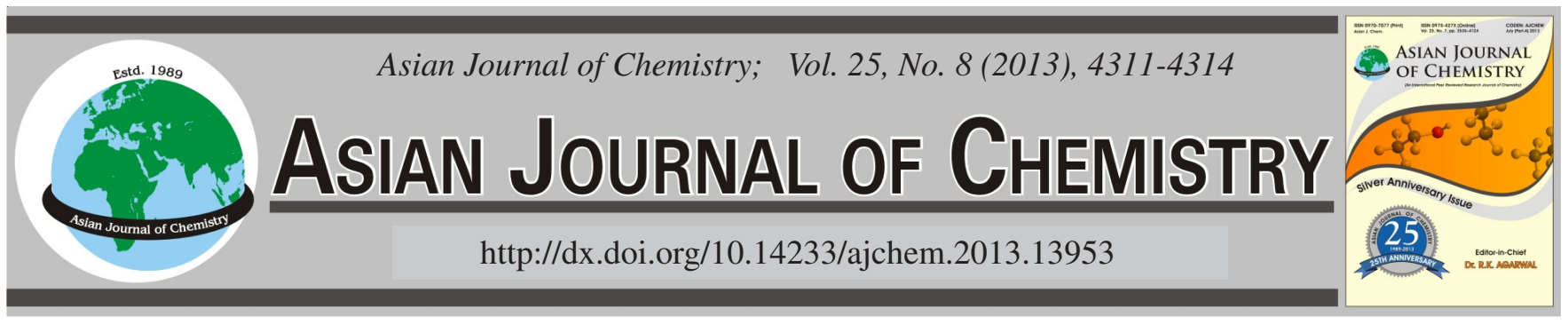

\title{
Kinetics and Mechanism of meso-Tetraphenylporphyriniron(III) Chloride Catalyzed Oxidation of Indole by Peroxomonosulphate
}

\author{
D. Kungumathilagam and K. Karunakaran*
}

Department of Chemistry, Sona College of Technology, Salem-636 005, India

*Corresponding author: E-mail: thilagamchem@gmail.com; drkk@ sonatech.ac.in

Mechanistic study on meso-tetraphenylporphyriniron(III) chloride (TPP) catalyzed oxidation of indole by peroxomonosulphate in aqueous acetonitrile medium have been carried out. The reaction follows a fractional order $(0.57)$ with respect to substrate and first order with respect to oxidant. The order with respect to catalyst was found to be one. Increase in percentage of acetonitrile decreased the rate of the reaction. The added $\mathrm{H}^{+}$did not affect the reaction rate. The reaction fails to initiate polymerization and free radical mechanism is ruled out. Activation and thermodynamic parameters have been computed. Nucleophilic attack of the ethylenic bond on the persulphate oxygen in presence of mesotetraphenylporphyriniron(III) chloride is envisaged to explain the reactivity. A suitable kinetic scheme based on the observations was proposed. Significant catalytic activity is observed for the reaction system in the presence of meso-tetraphenylporphyriniron(III) chloride.

Key Words: Indole, Peroxomonosulphate, Meso-tetraphenylporphyriniron(III) chloride, Kinetics, Oxidation.

ᄂ - - - - - - - - - - - - - - - - - - - - - - - - - - - -

\section{INTRODUCTION}

Indole, an electron-rich heteroaromatic nitrogen compound, is found in many naturally occurring compounds such as various plant alkaloids and fungal metabolites ${ }^{1,2}$ usually as metabolites of tryptophan. Indole, on oxidation gives products which are involved in significant biological processes ${ }^{3}$. Indoles possess significant biological activity ${ }^{4,5}$. Lawson and Witkop ${ }^{6}$ as well as Finch and Taylor ${ }^{7}$ have shown that N-bromosuccinimide can be used to convert indoles to oxindoles. Rangappa et al. ${ }^{8}$ have examined the kinetics of oxidation of indole by chloramines-T in the presence of Os(VIII) in alkaline medium. Peroxomonosulphate can also used to convert indoles to oxindoles ${ }^{9}$.

Peroxomonosulphate (PMS) ion, $\mathrm{HSO}_{5}^{-}$, is an anionic oxidant which is usually represented as oxone, $2 \mathrm{KHSO}_{5} \cdot \mathrm{KHSO}_{4} \cdot \mathrm{K}_{2} \mathrm{SO}_{4}$. Studies on the oxidation of organic compounds and inorganic complexes by PMS have been reported ${ }^{10}$. The synthetic utility of potassium peroxomonosulphate as an oxidant has been studied in detail ${ }^{11}$. It is a powerful oxidant and oxidizes alcohols, ketones, carboxylic acids, alkenes, arenes, phenols, amines and sulfides ${ }^{12,13}$. It hydroxylates alkanes and aromatic compounds ${ }^{14}$. The peroxo anion oxidation of indole has been carried out because its distinct reactivity is appropriate for an independent and precise kinetic study on the steps of the mechanism ${ }^{15}$.

Oxidation of organic compounds catalyzed by synthetic metalloporphyrin complexes has been one of the hot areas in chemistry during the last decade ${ }^{16}$. The metalloporphyrins can catalyze the oxidation of a variety of organic substrates with many different terminal oxidants ${ }^{16,17}$. The catalytic properties of the transition metal porphyrins are due to the fact that an oxo-transition metal porphyrin intermediate is formed, which can transfer the oxygen atom to a substrate or can accept an electron from the substrate ${ }^{16}$. Many stable oxo-transition metal complexes are known and have been characterized, but several of these complexes are inert and non-reactive as oxygen transfer reagents. Groves and coworkers ${ }^{18}$ described the use of meso-tetraphenylporphyriniron(III) chloride (TPP) in combination with the lipophilic iodosylbenzene, first used in vivo by Ullrich, for the epoxidation of olefins and the hydroxylation of alkanes.

Literature survey reveals that no report was available on the kinetics of oxidation of meso-tetraphenylporphyriniron(III) chloride catalyzed oxidation of indole, hence we have carried out this work with peroxomonosulphate.

\section{EXPERIMENTAL}

All the chemicals and solvents used were of analytical grade (Merck, India). Indole, oxone and meso-tetraphenylporphyriniron(III) chloride (TPP) (Sigma Aldrich) were used as such. All the solutions used in the study were made by using doubly distilled water. All the reagents were prepared freshly and used in the reaction. All the reactions were carried out in a thermostat and the temperature was controlled to $\pm 0.1^{\circ} \mathrm{C}$. 
Kinetic measurements: The kinetic studies were carried out in an aqueous acetonitrile medium. The reactions were performed by maintaining a large excess of [indole] over [PMS]. The reaction mixture was homogeneous throughout the course of the reaction. The reaction's progress was monitored for atleast two half-lives by iodometric estimation of unchanged oxidant at regular time intervals. The rate constants $\left(\mathrm{k}_{\mathrm{obs}}\right)$ were evaluated from the slopes of linear plots of $\log$ [titre] versus time.

Stoichiometry: Solutions of indole containing an excess of PMS were kept overnight at room temperature. Titrimetric estimation of the concentration of PMS consumed and assuming that all the indole taken had reacted, the stoichiometry of indole:PMS was found to be $1: 1$.

Product analysis: The reaction mixture after $48 \mathrm{~h}$, was extracted with chloroform, dried over anhydrous $\mathrm{Na}_{2} \mathrm{SO}_{4}$ and then evaporated. The residual brown solid obtained was subjected to column chromatography using silica gel and eluted with benzene and then evaporated. The melting point of the compound was found to be $116-118{ }^{\circ} \mathrm{C}$ confirms oxindole (literature value is $118-120{ }^{\circ} \mathrm{C}$ ) as the main product of the reaction. The product was also confirmed by UV-visible absorption spectra at $\lambda_{\max }$ of $397 \mathrm{~nm}$ (Fig. 7) which is in agreement with literature values.

Data analysis: Correlation studies were carried out using Microcal origin (version 6) computer software. The goodness of the fit was discussed using the correlation coefficient, $r$, in the case of simple linear regression and $\mathrm{R}$ in the case of multiple linear regressions.

\section{RESULTS AND DISCUSSION}

Factors influencing the rate of TPP catalyzed oxidation of indole by peroxomonosulphate such as [indole], [PMS], [TPP $],\left[\mathrm{H}^{+}\right]$and dielectric constant have been studied. Rate and activation parameters were evaluated.

Effect of [indole]: At a constant [PMS], [TPP], $\left[\mathrm{H}^{+}\right]$and fixed percentage of acetonitrile, kinetic runs were carried out with various initial concentrations of indole, which yielded rate constants whose values depended on [indole]. The pseudofirst order rate constants $\left(\mathrm{k}_{\mathrm{obs}}\right)$ thus obtained were found to increase with [indole] (Table-1) over a range of [indole] used $\left(3.0-7.0 \times 10^{-2} \mathrm{~mol} \mathrm{dm}^{-3}\right)$. The plot (Fig. 1) of $\log \mathrm{k}_{\mathrm{obs}}$ versus $\log$ [indole] is linear with a slope of 0.57 showing that the reaction is fractional order in [indole]. The plot (Fig. 2) of $1 / \mathrm{k}_{\text {obs }}$ versus $1 /$ [indole] is linear with definite intercept on the rate ordinate, which indicates the operation of MichalisMenton mechanism, where $\mathrm{K}_{3}$ is the equilibrium constant for the formation of Michalis-Menton complex and $\mathrm{k}_{4}$ the rate constant for the decomposition of Michalis-Menton complex. Usually first step is a fast pre-equilibrium and the electron transfer step is rate determining ${ }^{19}$.

Effect of [PMS]: The order of the reaction with respect to PMS was to be strictly unity as shown by the linearity of $\log$ [PMS] versus time plots, over $80 \%$ of the reaction. Further, the reaction rate decreases with increase in the concentration of oxidant, [PMS] $\left(1.5-3.5 \times 10^{-3} \mathrm{~mol} \mathrm{dm}^{-3}\right)$ at fixed [indole], [TPP $],\left[\mathrm{H}^{+}\right]$and percentage of acetonitrile (Table-1).

Effect of [TPP]: At a constant [indole], $[\mathrm{PMS}],\left[\mathrm{H}^{+}\right]$and fixed percentage of acetonitrile, kinetic runs were carried out
TABLE-1

PSEUDO-FIRST ORDER RATE CONSTANTS FOR THE TPP CATALYSED OXIDATION OF INDOLE BY PEROXOMONOSULPHATE AT $301 \mathrm{~K}$

\begin{tabular}{ccccc}
\hline $\begin{array}{c}\text { Indole] } \times 10^{2} \\
\left(\mathrm{~mol} \mathrm{dm}^{-3}\right)\end{array}$ & $\begin{array}{c}{[\mathrm{PMS}] \times 10^{3}} \\
\left(\mathrm{~mol} \mathrm{dm}^{-3}\right)\end{array}$ & $\begin{array}{c}{[\mathrm{TPP}] \times 10^{7}} \\
\left(\mathrm{~mol} \mathrm{dm}^{-3}\right)\end{array}$ & $\begin{array}{c}\mathrm{CH}_{3} \mathrm{CN} \\
\%(\mathrm{~V} / \mathrm{V})\end{array}$ & $\begin{array}{c}\mathrm{k}_{\mathrm{obs}} \times 10^{4} \\
\left(\mathrm{~s}^{-1}\right)\end{array}$ \\
\hline 3.0 & 2.5 & 4.0 & 80 & 1.52 \\
4.0 & 2.5 & 4.0 & 80 & 1.70 \\
5.0 & 2.5 & 4.0 & 80 & 2.09 \\
6.0 & 2.5 & 4.0 & 80 & 2.17 \\
7.0 & 2.5 & 4.0 & 80 & 2.45 \\
5.0 & 1.5 & 4.0 & 80 & 2.62 \\
5.0 & 2.0 & 4.0 & 80 & 2.42 \\
5.0 & 3.0 & 4.0 & 80 & 1.01 \\
5.0 & 3.5 & 4.0 & 80 & 0.82 \\
5.0 & 2.5 & 2.0 & 80 & 0.59 \\
5.0 & 2.5 & 6.0 & 80 & 3.20 \\
5.0 & 2.5 & 8.0 & 80 & 4.50 \\
5.0 & 2.5 & 10.0 & 80 & 7.81 \\
\hline
\end{tabular}

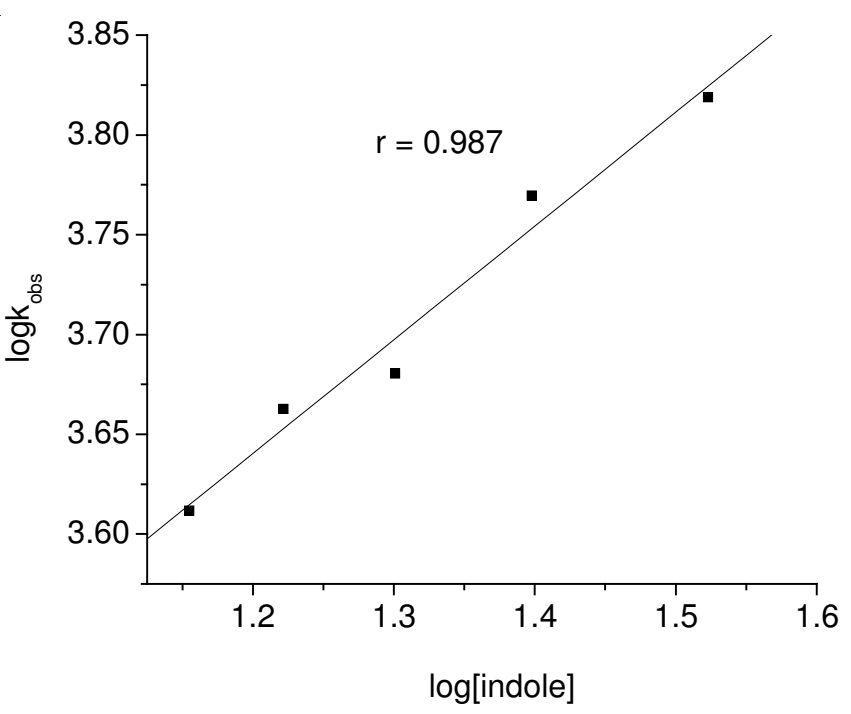

Fig. 1. Plot of $\log \mathrm{k}_{\mathrm{obs}}$ versus $\log$ [indole] for TPP catalyzed oxidation of indole by peroxomonosulphate in acetonitrile medium

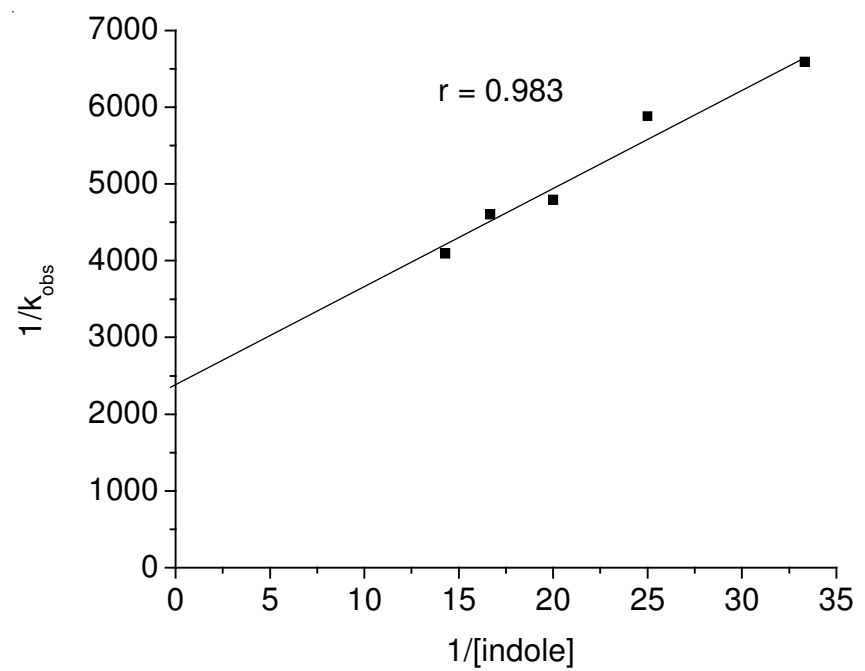

Fig. 2. Plot of $1 / \mathrm{k}_{\mathrm{obs}}$ versus $1 /$ [indole] for TPP catalyzed oxidation of indole by peroxomonosulphate in acetonitrile medium

with various initial concentrations of [TPP], which yielded rate constants whose values depended on [TPP]. The pseudofirst order rate constants $\left(\mathrm{k}_{\mathrm{obs}}\right)$ thus obtained were found to increase with [TPP] (Table-1) over a range of [TPP] used (0.2- 
$\left.1.0 \times 10^{-7} \mathrm{~mol} \mathrm{dm}^{-3}\right)$. A linear plot was obtained between logkobs and $\log$ [TPP] (Fig. 3) with a slope of 1.07, indicating that the order of the reaction with respect to catalyst was one.

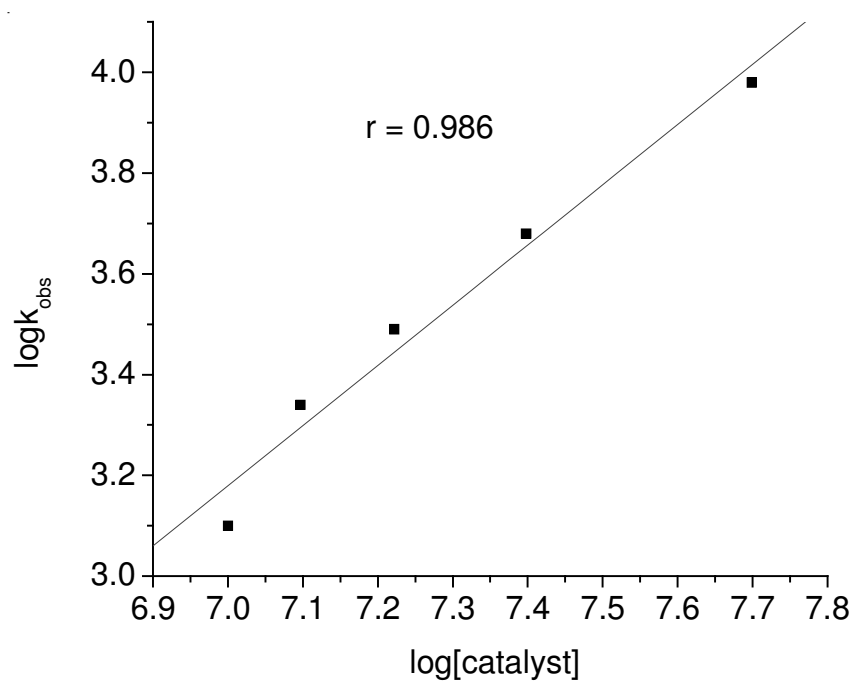

Fig. 3. Plot of $\log \mathrm{k}_{\mathrm{obs}}$ versus $\log$ [catalyst] showing the effect of catalyst concentration reaction rate

Effect of $\left[\mathbf{H}^{+}\right]$: The reaction rates measured with various $\left[\mathrm{H}^{+}\right]\left(0.08-0.40 \mathrm{~mol} \mathrm{dm}^{-3}\right)$ and were found to be the same (Table-2). Such kinetic behaviour indicates the nonexistence of any protonation equilibrium with respect to both PMS and indole in the experiment.

TABLE-2

EFFECT OF $\left[\mathrm{H}^{+}\right]$CONCENTRATION ON THE REACTION RATE

$\begin{array}{cc}{\left[\mathrm{H}^{+}\right] \times 10^{2}\left(\mathrm{~mol} \mathrm{dm}^{-3}\right)} & \mathrm{k}_{\mathrm{obs}} \times 10^{4}\left(\mathrm{~s}^{-1}\right) \\ 8.0 & 3.40 \\ 16.0 & 3.06 \\ 24.0 & 3.31 \\ 32.0 & 3.46 \\ 40.0 & 3.93\end{array}$

$[\mathrm{PMS}]=2.5 \times 10^{-3} \mathrm{~mol} \mathrm{dm}{ }^{-3}$, indole $]=5.0 \times 10^{-2} \mathrm{~mol} \mathrm{dm}^{-3},[\mathrm{TPP}]=$ $4.0 \times 10^{-7} \mathrm{~mol} \mathrm{dm}^{-3}$, acetonitrile: water $=40: 10$, temperature $=301 \mathrm{~K}$.

Effect of dielectric constant: In order to determine the effect of dielectric constant (polarity) of the medium on rate, the oxidation of indole by peroxomonosulphate was studied in aqueous acetonitrile mixtures of various compositions (Table-3). The data clearly reveals that the rate increases with decrease in the percentage of acetonitrile, i.e., with increasing dielectric constant or polarity of the medium and leads to the influence that there is a charge development in the transition state involving a more polar activated complex than the reactants ${ }^{20,21}$, a neutral molecule [indole] and a mono negative ion $\left(\mathrm{HSO}_{5}^{-}\right)$suggesting a polar (ionic) mechanism.

Test for free radical intermediates: No turbidity was observed when a freshly distilled acrylonitrile monomer was added to the deaerated reaction mixture indicating the absence of free radical intermediates.

Rate and activation parameters: The effect of temperature was studied in the range of 301-323 $\mathrm{K}$ and the results were shown in Table- 4 . The Arrhenius plot of $\ln \mathrm{k}_{\mathrm{obs}} / \mathrm{T}$ versus $1 / \mathrm{T}$ was found to be linear (Fig. 4). The value of energy of activation $\left(\mathrm{E}_{\mathrm{a}}\right)$ was found to be $10.36 \mathrm{~kJ} \mathrm{~mol}^{-1} \mathrm{~K}^{-1}$ and $\Delta \mathrm{H}^{\#}=$
TABLE-3

EFFECT OF DIELECTRIC CONSTANT ON THE REACTION RATE AT $301 \mathrm{~K}$

\begin{tabular}{ccc}
\hline $\mathrm{CH}_{3} \mathrm{CN}: \mathrm{H}_{2} \mathrm{O}$ & $\mathrm{D}^{\mathrm{a}}$ & $\mathrm{k}_{\text {obs }} \times 10^{4}\left(\mathrm{~s}^{-1}\right)$ \\
\hline $90: 10$ & 41.47 & 0.31 \\
$80: 20$ & 45.48 & 2.09 \\
$70: 30$ & 49.71 & 2.76 \\
$60: 40$ & 53.83 & 5.42 \\
$50: 50$ & 57.95 & 8.95 \\
\hline$[\mathrm{PMS}]=2.5 \times 10^{-3} \mathrm{~mol} \mathrm{dm}^{-3}$, [indole] $=5.0 \times 10^{-2} \mathrm{~mol} \mathrm{dm}^{-3},[\mathrm{TPP}]=4.0$ \\
$\times 10^{-7} \mathrm{~mol} \mathrm{dm}^{-3}$. ${ }^{a}$ Values are calculated from the values of pure solvent.
\end{tabular}

TABLE-4

EFFECT OF TEMPERATURE ON THE REACTION RATE

\begin{tabular}{llllll}
\hline Temp. $(\mathrm{K})$ & 301 & 306 & 312 & 318 & 323 \\
\hline $\mathrm{k}_{\mathrm{obs}} \times 10^{4}\left(\mathrm{~s}^{-1}\right)$ & 1.86 & 2.09 & 3.34 & 4.66 & 5.73 \\
\hline$[\mathrm{PMS}]=2.5 \times 10^{-3} \mathrm{~mol} \mathrm{dm}^{-3}$, [indole] & $=5.0 \times 10^{-2} \mathrm{~mol} \mathrm{dm}^{-3},[\mathrm{TPP}]=$ \\
$4.0 \times 10^{-7} \mathrm{~mol} \mathrm{dm}^{-3}$. Acetonitrile:water $=$ & $40: 10$.
\end{tabular}

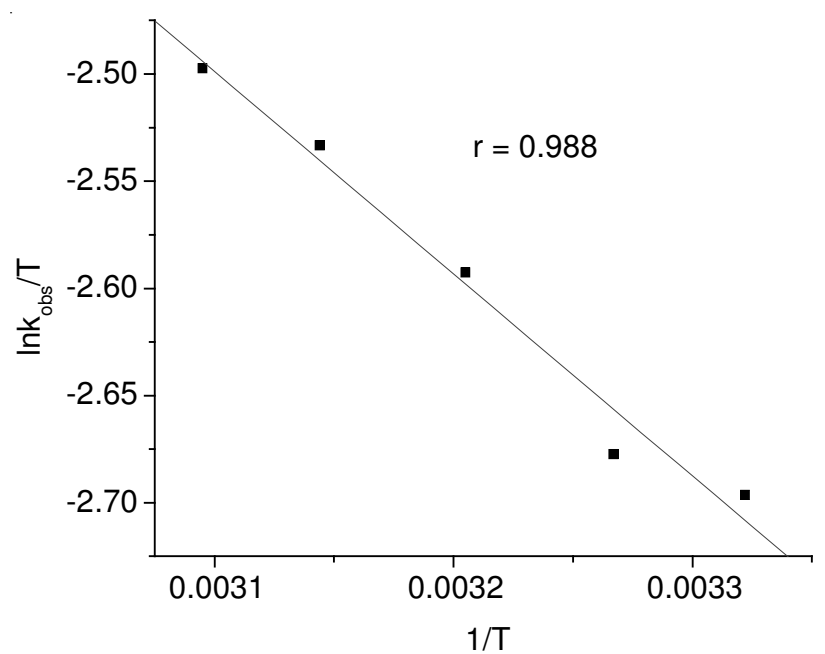

Fig. 4. Plot of $\ln \mathrm{k}_{\mathrm{obs}} / \mathrm{T}$ versus $1 / \mathrm{T}$ showing the effect of temperature on reaction rate

$7.84 \mathrm{~kJ} \mathrm{~mol}^{-1}, \Delta \mathrm{S}^{\#}=-194.10 \mathrm{~J} \mathrm{~K}^{-1} \mathrm{~mol}^{-1}, \Delta \mathrm{G}^{\#}=66.65 \mathrm{~kJ} \mathrm{~mol}^{-1}$. The large negative value of entropy of activation $\left(\Delta \mathrm{S}^{\#}\right)$ obtained is attributed to the severe restriction of solvent molecules around the transition state ${ }^{22}$.

Mechanism: Peroxomonosulphate exists ${ }^{23}$ as $\mathrm{HSO}_{5}{ }^{-}$in acidic solution. Although many peroxy anions are effective nucleophiles. $\mathrm{HSO}_{5}{ }^{-}$is weak nucleophile ${ }^{24}$. Inspite of the fact that free radicals can arise from the facile homolysis of the oxygen-oxygen bond ${ }^{25}$, an ionic mechanism is favoured in certain reactions involving oxidations by peroxides. In the present investigation no observed polymerization in the presence of acrylonitrile rules out a free radical process. Hence ionic mechanism was favoured in this study. Generally, the enhancement of the electrophilic activity of peroxide will minimize the importance of undesirable free radical pathways, resulting in a mixture of products ${ }^{26}$. The Michaelis-Menton dependence of the oxidation rate on [indole] confirms the formation of complex in a rapid pre-equilibrium.

The first step is the formation of a complex between oxone and TPP which was confirmed from the UV-visible spectra (Fig. 5). This complex further decomposed and give Por-Fe(V) $=\mathrm{O}$ is in agreement with the literature study ${ }^{27}$. This Por-Fe(V) $=\mathrm{O}$ may further react with the indole to form a complex (Fig. 6) 


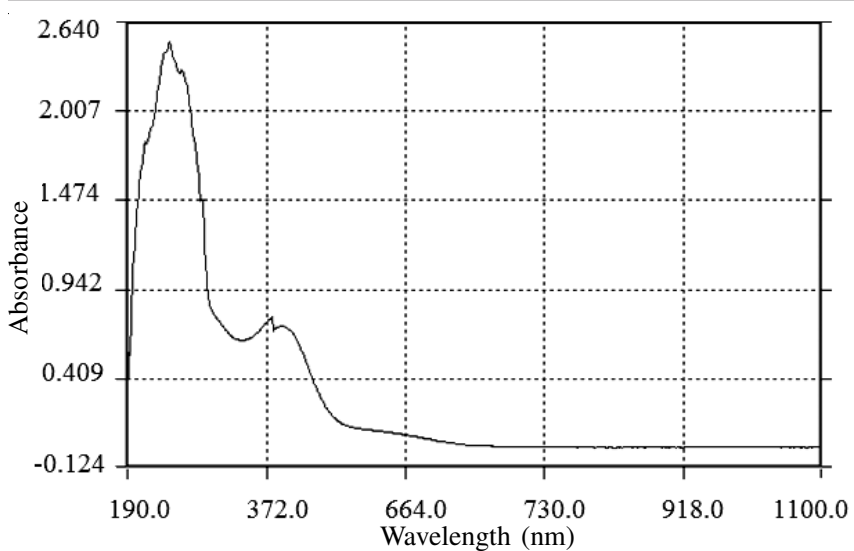

Fig. 5. UV spectrum showing the complex formation between TPP and PMS at $237 \mathrm{~nm}$

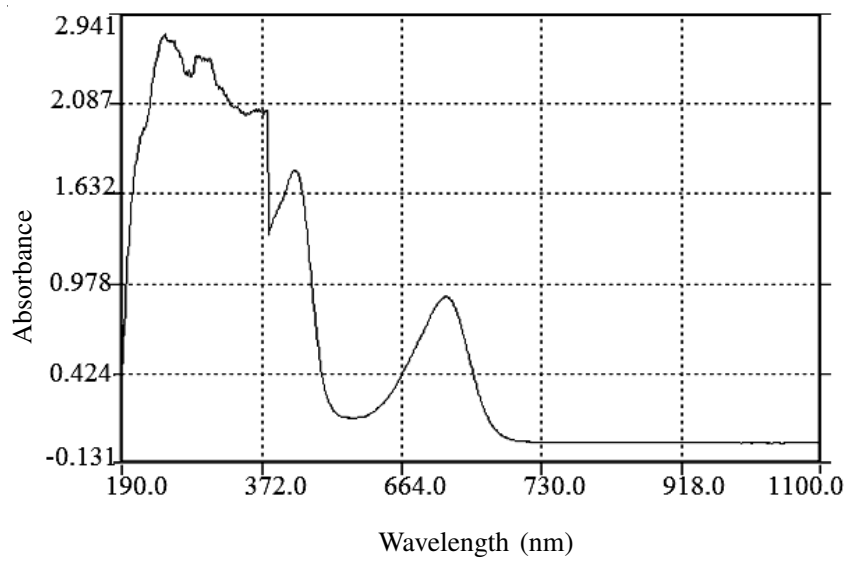

Fig. 6. UV spectrum showing formation of intermediate complex at 694 $\mathrm{nm}$ between indole and $\operatorname{PorFe}(\mathrm{V})=\mathrm{O}$

at $694 \mathrm{~nm}$, which would give the product in the next step (Fig. 7). This type of product was already reported ${ }^{9}$. The oxygen transfer step is associated with large negative value of entropies of activation and significant enthalpies of activation. The catalytic activity of TPP is significant and this conversion exhibits first order. The plot of $\log$ [catalyst] versus $\log \mathrm{k}_{\mathrm{obs}}$ is found to be linear. In accordance with the above observations and stoichiometry of the reaction, the following reactions are involved to constitute the most probable mechanism of the reaction (Scheme-I).

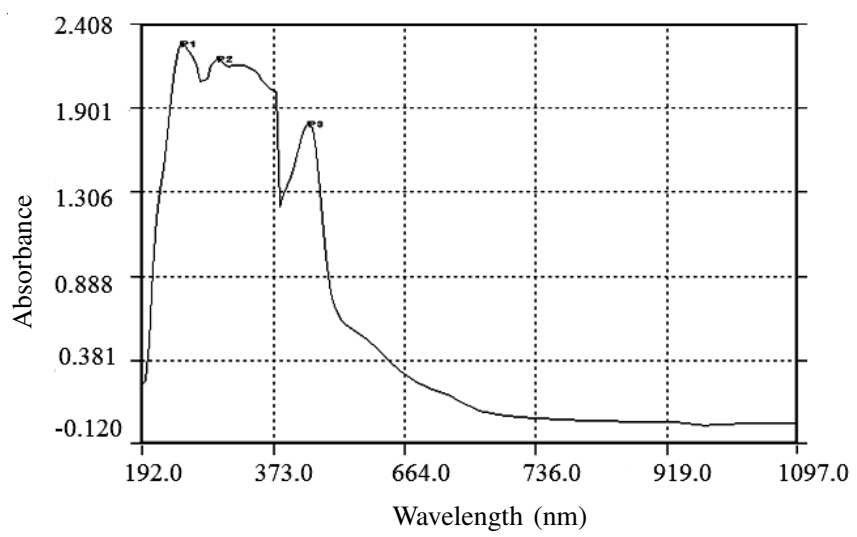

Fig. 7. UV spectrum showing the formation of product at $397 \mathrm{~nm}$ after the decomposition of intermediate complex at $694 \mathrm{~nm}$

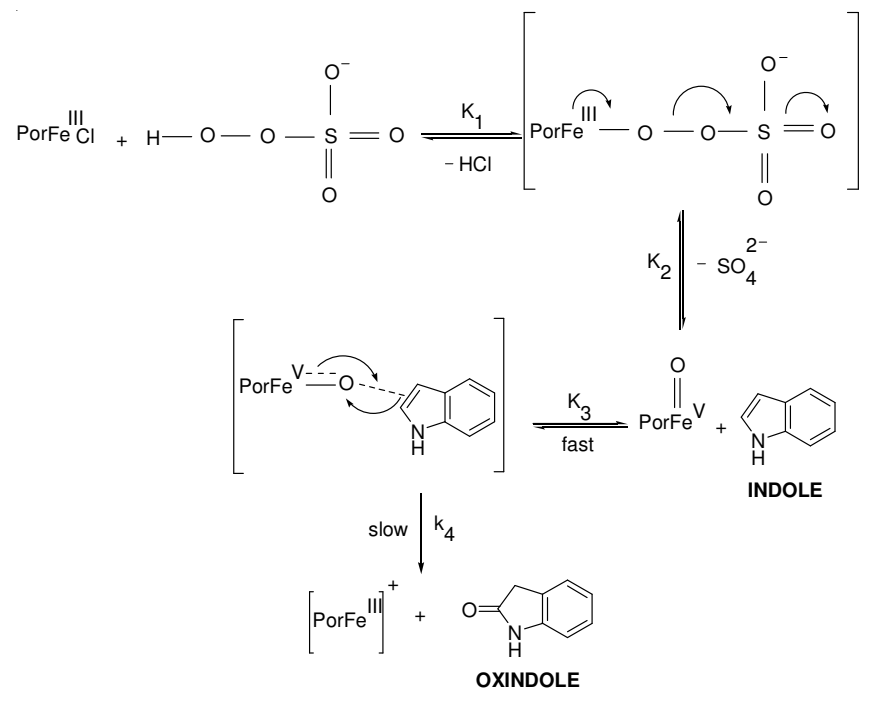

Scheme-I: $\quad$ Probable mechanism for the meso-tetraphenyl porphyriniron(III) chloride catalysed oxidation of indole by peroxomonosulphate

\section{REFERENCES}

1. R.J. Sundberg, Indole, Kirk-othmer Encyclopedia of Chemical Technology, Wiley, New York (1995).

2. R.N. Goyal and A. Sangal, Electrochim. Acta, 50, 2135 (2005).

3. A.T. Al-kazwini, P. O'Neill, G.E. Adams, R.B. Cundall, G. Lang and A. Junino, J. Chem. Soc. Perkin Trans II, 1941 (1991).

4. A.T. Al-kazwini, P. O'Neill, G.E. Adams, R.B. Cundall, A. Junino and J. Maignan, J. Chem. Soc. Perkin Trans. II, 657 (1992).

5. S.N. Krylov and H.B. Dunford, J. Phys. Chem., 100, 913 (1996).

6. W.B. Lawson and B. Witkop, J. Org. Chem., 26, 263 (1961).

7. N. Finch and W.E. Taylor, J. Am. Chem. Soc., 84, 3871 (1962).

8. K.S. Rangappa, D.T. Esterline, C.K. Mythily, D.S. Mahadevappa and S.Y. Ambedkar, Polyhedron, 12, 1719 (1993).

9. S.P. Meenakshisundaram and N. Sarathi, Int. J. Chem. Kinet., 39, 46 (2007).

10. (a) R. Somuthevan, R. Renganathan and P. Maruthamuthu, Inorg. Chim. Acta, 45, L165 (1980); (b) A. Jameel and P. Maruthamuthu, J. Indian Chem. Soc., 75, 368 (1998).

11. R. Suthakaran, P. Subramaniam and C. Srinivasan, Proc. Indian Acad. Sci., 97, 555 (1986).

12. R.J. Kennedy and A.M. Stock, J. Org. Chem., 25, 1901 (1960).

13. B.M. Trost and D.P. Curran, Tetrahedron Lett., 22, 1287 (1981).

14. R. Mello, M. Fiorentino, C. Fusco and R. Curci, J. Am. Chem. Soc., 111, 6749 (1989)

15. M. Balon, M. Munoz, P. Guardado, J. Hidalgo and C. Carmona, Org. Chem., 58, 7469 (1993).

16. B. Meunier, Chem. Rev., 92, 1411 (1992).

17. J. Larsen and K. Anker-Jorgensen, J. Chem. Soc. Perkin Trans II, 1213 (1992).

18. J.T. Groves, T.E. Nemo and R.S. Myers, J. Am. Chem. Soc., 101, 1032 (1979).

19. D.S. Bhuvaneshwari and K.P. Elango, J. Indian Chem. Soc., 86, 242 (2009).

20. K.J. Laidler, Chemical Kinetics, Tata-McGraw Hill, New Delhi (1965).

21. F. Ruff and A. Kucsman, J. Chem. Soc. Perkin Trans. II, 683 (1985).

22. S.P. Meenakshisundaram, M. Selvaraju, N.M. Made Gowda and K.S. Rangappa, Int. J. Chem. Kinet., 37, 649 (2005).

23. M.S. Ramachandran, T.S. Vivekanadam and V. Arunachalam, Bull. Chem. Soc. (Japan), 59, 1549 (1986).

24. D.M. Davis and M.E. Deary, J. Chem. Soc. Perkin Trans II, 559 (1992).

25. J.A. Kerr, Chem. Rev., 66, 465 (1966).

26. Z. Zhu and J.H. Espenson, J. Org. Chem., 60, 1326 (1995).

27. X.-T. Zhou, H.-B. Ji and Q.-L. Yuan, J. Porphyr. Phthalocya., 12, 94 (2008). 